\title{
Editorial
}

\section{Fluctuation-Enhanced Sensing}

\author{
Graziella Scandurra $\left(\mathbb{D},{ }^{1}\right.$ Janusz Smulko $\mathbb{D}^{2},{ }^{2}$ and Laszlo B. Kish $\mathbb{D}^{3}$ \\ ${ }^{1}$ Department of Engineering, University of Messina, Messina I-98166, Italy \\ ${ }^{2}$ Department of Metrology and Optoelectronics, Gdańsk University of Technology, Gdańsk 80-233, Poland \\ ${ }^{3}$ Department of Electrical and Computer Engineering, Texas A\&M University, College Station, TX 77843-3128, USA
}

Correspondence should be addressed to Graziella Scandurra; gscandurra@unime.it

Received 9 July 2020; Accepted 9 July 2020; Published 23 November 2020

Copyright (c) 2020 Graziella Scandurra et al. This is an open access article distributed under the Creative Commons Attribution License, which permits unrestricted use, distribution, and reproduction in any medium, provided the original work is properly cited.

Fluctuation-enhanced sensing (FES) is an exciting and relatively new research field that promises to extend the range of information that can be extracted from a single sensor. In FES, the stochastic fluctuations of the sensor signal, rather than its average value, are recorded and analyzed. Typical components of such fluctuations are due to interactions at the microscopic level. Proper statistical analysis provides optimum sensory information.

FES was used for the first time in the 90s, but it has not been commercially available yet because of the relatively limited amount of test data and the requirement of a special measurement setup. Sensor interface and instrumentation in a FES system have to be specifically designed to extract and amplify the low-frequency stochastic signal components, which are usually orders of magnitude weaker than the classical, deterministic sensor signal. Then, selected statistical properties of the amplified noise are analyzed in order to generate a corresponding pattern as a "stochastic fingerprint" of the sensed agent. The power spectral density of the noise signal is often used as an output pattern, but FES has been proven effective with higher-order statistics and other more advanced methods as well.

Many open questions must be addressed before FES can become a commercially available technique. Such issues include the design of highly sensitive instrumentation and the most effective data analysis, the development of working physical models for the interpretation of the stochastic data involving the sensor structure and sensing mechanisms, the selection of proper materials and structures, and finding sensing configuration for optimized sensitivity and selectivity.
Despite the challenges, FES exhibits a sensitivity of orders of magnitude higher than that of conventional electronic noses and tongues. Moreover, a single sensor can act as a complex high-dimensional electronic nose or tongue.

Therefore, it is important to enhance research activities and interactions in this subject. The purpose of this special issue is to turn the attention to the potentials of FES and to bring specialists together to reach the critical mass for a breakthrough in this field.

Because of the complexity of the topic, special attention was given to the papers including experimental data or results actually relevant for the future development of FES. After a rigorous peer-review process, 4 papers were accepted for publication in this special issue.

The paper by M. Piotto et al. proposes an original approach to model the noise density in sensors based on a single hot wire or pairs of thermally coupled wires. Since hot wires are used in a large variety of sensing devices and instruments, this study could give an important contribution to advanced sensor design. The model presented in this paper consists of an original combination of a previous electrothermal model of the wire with well-established assumptions on the thermal noise in conductors that carry moderate current densities. The model parameters can be easily determined by means of small-signal impedance measurements as a function of frequency. The effects of the electrothermal feedback of both hot wires and hot-wire pairs on flicker noise are also intrinsically covered by the proposed approach. The results showed in this work should contribute to widen the knowledge of noise in out-of-equilibrium electrical systems. 
The paper by M. Macucci and P. Marconcini is focused on the noise properties of graphene, which is one of the principal materials for the realization of modern sensors whose ultimate performance is often right determined by the flicker noise of graphene. Indeed, graphene exhibits, compared to the vast majority of ordinary semiconductors, a peculiar behaviour of the flicker noise power spectral density as a function of the charge carrier density. In this paper, this difference is addressed and explained, and a comparison between graphene and other semiconducting materials is carried out. The conducted analysis, which clarifies the mechanisms that cause or prevent a reduction, at proper bias conditions, of the intrinsic sensor flicker noise, can be useful for the design of low-noise devices and in particular of highsensitivity sensors.

The paper by G. Scandurra et al. proposes a new approach for the extraction of the equivalent parameters of quartz tuning forks (QTF) used as sensors by means of noise measurements. By means of spectral analysis, noise is used as the test signal for the determination of the frequency response of a circuit including the quartz tuning fork whose parameters need to be determined. A new approach for the analysis of strongly peaked noise spectra was developed in order to allow the correct measurement of the strongly peaked noise spectrum at the output of the system, which is the result of the high-quality factor of any quartz tuning fork-based sensor. With the proposed approach, the best compromise in terms of accuracy and measurement time can be obtained in a single measurement run. The ability to accurately estimate the PSD over a narrow bandwidth across the resonance frequency of a QTF-based sensor may facilitate the exploration of fluctuation-enhanced sensing (FES) with QTF-based sensors.

The paper by J. Smulko et al. presents experimental results of fluctuation-enhanced gas sensing by low-cost resistive sensors based on a mixture of graphene flakes and $\mathrm{TiO}_{2}$ nanoparticles, both photocatalytic and activated by UV light. The sensors' response to two UV LEDs of different wavelengths was observed in an ambient atmosphere of synthetic air and toxic $\mathrm{NO}_{2}$ at selected concentrations. It was observed that flicker noise changes its frequency dependence at different UV light wavelengths, thereby providing additional information about the ambient atmosphere, and that the power spectral density changes by a few times as a result of UV light irradiation. The sensors were operated at different temperatures, and the effect of UV light on gas sensing was most apparent at low operating temperature. In conclusion, UV light activates the gas sensing layer and improves gas detection at low concentrations of $\mathrm{NO}_{2}$. This result is desirable for the detection of the components of gas mixtures, and the modulated sensor can replace an array of independent resistive sensors which would consume much more energy for heating. It is also suggested in the paper that a more advanced technology for preparing the gas sensing layer, by use of spin coating, will produce corresponding layers with thickness of about a few $\mu \mathrm{m}$, which is about ten times less than that for the tested samples, and consequently, the effects induced by the applied UV light, having a penetration depth of only a few $\mu \mathrm{m}$, would then be amplified. The presented results open a new perspective on enhanced gas sensing for emerging gas sensing materials.

Graziella Scandurra

Janusz Smulko

Laszlo B. Kish

\section{Conflicts of Interest}

The authors declare that there is no conflict of interest regarding the publication of this paper. 